\title{
Caracterização dos educadores de infância angolanos: envolvimento, autoconceito e necessidades de formação
}

\author{
Genoveva A. Borges ${ }^{1}$, Feliciano H. Veiga ${ }^{1}$ \\ gumartins6@hotmail.com, fhveiga@ie.ulisboa.pt \\ ${ }^{1}$ Instituto de Educação da Universidade de Lisboa, Portugal
}

\begin{abstract}
Resumo
O interesse pelo estudo do envolvimento, do autoconceito e da formação dos educadores de infância é visto como essencial na educação, pelo facto de tais variáveis poderem ter fortes influências no desenvolvimento psicossocial das crianças em idade pré-escolar. Os educadores de infância devem assegurar às crianças, além do ensino, modelos positivos de comportamento para um desenvolvimento global, o que requer condições pessoais e profissionais benéficas aos educadores. A presente investigação teve por objetivo caracterizar o envolvimento, o autoconceito e as necessidades de formação dos educadores de infância em Angola, ou seja, saber como se distribuem os educadores pelas dimensões do envolvimento no trabalho, do autoconceito profissional e, ainda, das necessidades de formação, em termos de baixa versus alta autoavaliação. A amostra foi constituída por 198 educadores de infância em serviço efetivo, em infantários de três províncias angolanas - Benguela, Cuanza-Sul e Luanda. Utilizaram-se três escalas, a Escala de Avaliação do Envolvimento, a Escala de Avaliação do Autoconceito e a Escala de Avaliação das Necessidades de Formação dos Educadores de Infância, esta com respostas em três níveis, desde nenhum interesse a interesse elevado, e as duas primeiras com respostas de concordância de 1 a 6 . A análise dos resultados permitiu encontrar que a maioria dos educadores de infância apresentou alta autoavaliação, quer no envolvimento no trabalho, quer no autoconceito profissional, quer nas necessidades de formação. No entanto, foi notória a quantidade de educadores com baixo envolvimento no trabalho, com baixo autoconceito profissional e com necessidades de formação apelativas. Explicações dos resultados e implicações para a formação dos educadores em Angola são também apresentadas.
\end{abstract}

Palavras-Chave: educadores de infância; envolvimento; autoconceito; necessidades de formação; Angola.

\begin{abstract}
Interest in the study of the engagement, self-concept and training of early childhood educators is seen as essential in education, because such variables may have strong influences on the psychosocial development of pre-school children. Early childhood educators should assure children, besides teaching, positive behavioural models for global development, which requires both personal and professional conditions that are beneficial to educators. The purpose of the present research was to characterize the engagement, self-concept and training needs of early childhood educators in Angola, this is, to know how children's educators are distributed through the dimensions of engagement in work, professional self-concept and training needs, in terms of low versus high self-assessment. The sample consisted of 198 full-time childhood educators in nurseries in three Angolan provinces - Benguela, Cuanza-Sul and Luanda. Three scales were used: the Engagement Evaluation Scale, the Self-Concept Assessment Scale, and the Infant Educators' Training Needs Assessment Scale, with responses at three levels, from no interest to high interest, and the first two with concordance responses from 1 to 6 . The analysis of the results allowed finding that the majority of the educators of childhood displayed high self-assessment, both in the engagement in the work, in the professional self-concept, and in training needs. However, the number of childhood educators with low engagement in work, with low professional self-concept and appealing training needs was notorious. Explanations of the results and implications for the training of educators in Angola are also presented.
\end{abstract}

Keywords: childhood educators; engagement; self-concept; training needs; Angola. 


\section{Introdução}

Vivemos numa sociedade que já não admite uma escola passiva, mas, sim, impõe uma escola ativa, empreendedora e com acesso ao meio, uma escola promotora de cultura de comunicação, de partilha com a comunidade escolar e com o meio envolvente, com o objetivo de contribuir para o desenvolvimento equilibrado dos indivíduos (Saraiva, 2002). Segundo o autor, por esse motivo, são impostos pela escola atual novos procedimentos, novos deveres a todos os que colaboram no processo de ensino e aprendizagem: professores, pais e encarregados de educação, entre outros. A função do professor, fundamental enquanto impulsor do processo, é decisiva para o êxito de qualquer reforma educativa. Assim, a escola deve ser um contexto de troca de saberes, onde todos os intervenientes convivem parte do tempo. Por essa razão, é essencial que cada um se sinta peça componente da mesma, pois que, pensar numa instituição como é o caso da escola, obriga necessariamente que se pense nos sujeitos que a integram, que laboram e participam na conquista de metas comuns e nas inter-relações que se criam. Lima (citado por Saraiva, 2002) destaca que, por meio de um sistema de combinação de quatro vertentes: democraticidade, regulamentação, envolvimento e orientação, é possível caracterizar as diversas maneiras e modelos de participação na escola. Nesse âmbito, o envolvimento determina um comportamento de maior ou de menor compromisso dos atores nas atividades de organização, de maneira a mostrar certos interesses e decisões. Por esse motivo, a participação dos sujeitos pode mostrar vigor/energia, prudência ou indiferença. Sendo próprio do ser humano interrogar-se acerca de quem é, o que sente e o que pensa sobre si próprio, existe uma via de acesso ao autoconceito, considerado como o conhecimento que o sujeito tem de si mesmo enquanto sujeito e de si-próprio em relação aos demais (Veiga, 2012). Quanto às necessidades de formação, esta referência é feita quando é realizada uma avaliação das dessas necessidades sempre que se procura reconhecer questões para as quais a formação tenha a possibilidade de colaborar (Barbier \& Lesne, 1986). Estes autores referem, ainda, que analisar necessidades é um exercício de edificação de objetivos num plano geral ou particular, precisando de ser observado tal como é. Realçam que não há um conjunto de processos de análise dessas necessidades, reciprocamente exclusivos mas sim complementares. Este facto justifica-se porque as mesmas necessidades de formação não compõem dados objetivos, uma vez que surgem do requerido institucionalmente (pelas questões laborais experienciadas no quotidiano).

Em Angola tem se observado uma grande oferta de serviços no Ensino Pré-escolar, em instituições educativas estatais e privadas, onde uma grande parte dos educadores de infância que aí trabalha não possui formação específica ao nível do ensino médio ou superior. Nesse sentido, o contexto educativo angolano e as situações vivenciadas nestas instituições, como a falta de atendimento especializado, levaram a colocar o seguinte problema de investigação: Quais as variações do envolvimento, do autoconceito profissional e das necessidades de formação dos educadores de infância de Angola, como se relacionam estes constructos entre si, e como oscilam em função de variáveis sociodemográficas, da formação inicial, da formação profissional, dos objetivos académicos e do grau de satisfação profissional? Este problema - e as questões que dele derivaram, a seguir apresentadas - emerge, não só de contactos mantidos com infantários de Angola e do interesse pessoal pelo envolvimento, autoconceito e pela formação dos mesmos, mas também da preocupação em procurar dar respostas aos problemas de cariz profissional dos educadores de infância.

\section{Metodologia}

\subsection{Método}

Esta pesquisa é de tipo quantitativo por se terem obtido os dados com recurso à uma inquérito por questionário cujo tratamento estatístico, permitiu alcançar algumas conclusões pertinentes (Richardson, citado por Dalfovo et al., 2008). Neste caso os dados foram sujeitos a análise estatística através da versão 23.0 do programa informático SPSS.

\subsection{Objetivos e questões de investigação}

É objetivo principal deste estudo caracterizar a amostra de educadores de infância relativamente ao envolvimento, ao autoconceito e às necessidades de formação. Assim, e com base no objetivo traçado, 
decidiu-se formular as questões que se seguem: Q1 - Como se distribuem os educadores de infância pelas dimensões do envolvimento no trabalho, em termos de envolvimento baixo versus alto? Q2 - Como se distribuem os educadores de infância pelas dimensões do autoconceito, em termos de autoconceito baixo versus alto? e Q3 - Como se distribuem os educadores de infância pelas dimensões das necessidades de formação, em termos de elevado, intermédio e nenhum interesse?, restringir-nosemos a análise dos resultados dessa distribuição, ao envolvimento, ao autoconceito e às necessidades de formação.

\subsection{Amostra}

A amostra deste estudo foi constituída por 198 educadores de infância dos quais 196 do género feminino (99\%) e 2 do género masculino (1\%), em serviço efetivo em infantários de três províncias de Angola (Benguela, Cuanza-sul e Luanda).

\subsection{Técnicas e instrumentos de investigação}

O instrumento de recolha de dados foi o inquérito por questionário constituído pelas escalas: Escala de Avaliação do Envolvimento dos Educadores de Infância 1 (EAE-EI 1), adaptada da versão brasileira da Utrecht Work Engagement Scale (UWES), de Angst, Benevides-Pereira e Porto-Martins (2009) com duas dimensões características (Vigor-dedicação e Absorção), e pela Escala de Avaliação do Envolvimento dos Educadores de Infância 2 (EAE-EI 2), adaptada da Escala de Representações dos Professores acerca dos seus Comportamentos de Cidadania Docente (ERP-CCID) de Fonseca (2009), com duas dimensões características (Interpessoalidade e Consciência pedagógica-participação), ambas para medir o envolvimento dos educadores.

$\mathrm{Na}$ avaliação do autoconceito utilizou-se a Escala de Avaliação do Autoconceito dos Educadores de Infância 1 (EAA-EI 1), adaptada de Veiga et al. (2006) com quatro dimensões características (Competência, Relações-iniciativas-riscos, Autoaceitação e Satisfação) e pela Escala de Avaliação do Autoconceito dos Educadores de Infância 2 (EAA-EI 2), também adaptada de Fonseca (2009), com duas dimensões características (Reconhecimento Profissional em Contexto Laboral e Pertençacooperatividade em Contexto Laboral).

$\mathrm{Na}$ avaliação das necessidades de formação a escala utilizada foi a Escala de Avaliação das Necessidades de Formação dos Educadores de Infância (EANF-EI), com três dimensões características (Gestão da profissão, Administração da progressão das Aprendizagens e Utilização das novas tecnologias), adaptada da Escala de Necessidades de Formação de Professores (ENFP) de Rocha (2010).

\subsection{Procedimento}

Antes da recolha dos dados realizou-se um estudo piloto em agosto de 2011 em que estiveram envolvidas 11 educadoras (1 portuguesa e 10 angolanas), com o objetivo de descobrir pontos fracos e potenciais problemas no inquérito, tais como interpretação errada de questões ou de termos e preenchimento incorreto como consequência da interpretação das questões, para que se pudesse resolver antes da sua aplicação definitiva. Este é um procedimento importante pela capacidade de atestar, medir, rever e aperfeiçoar os instrumentos e processos de investigação (Canhota, 2008; Mackey \& Gass, citados por Bailer, Tomitch \& D`Ely, 2011)

Após a elaboração do inquérito definitivo, dirigiu-se ao Sr. Ministro da Educação de Angola e ao Sr. Ministro da Assistência e Reinserção Social (MINARS) um pedido de autorização para a realização da pesquisa. O consentimento dos Srs. Ministros foi dado por escrito. Contactou-se a Direção municipal do MINARS para a cedência de um mapa informativo acerca da localização das instituições de Ensino Pré-escolar públicas e privadas inscritas e a funcionar efetivamente, sendo explicado o objetivo do estudo. Antes da distribuição dos questionários, solicitaram-se encontros com os Diretores pedagógicos dos jardins de infância constantes no mapa, com o objetivo de comunicar os fins do estudo. O mesmo procedimento foi tido em consideração nas outras províncias. Os inquéritos começaram a ser distribuídos pessoalmente em abril de 2012. Através da descrição dos objetivos pretendeu-se dar a 
conhecer a relevância da pesquisa e das respostas dos educadores para o desenvolvimento do estudo, garantindo-lhes a confidencialidade das respostas e o anonimato, o direito à privacidade e à não participação, assumindo-se que os dados seriam usados exclusivamente no âmbito da pesquisa.

\section{Resultados}

3.1 Como se distribuem os educadores de infância pelas dimensões do envolvimento no trabalho, em termos de envolvimento baixo versus alto?

Em resposta a esta questão de estudo, a maioria dos educadores de infância apresentou envolvimento alto, em todas as dimensões, exceto na dimensão Consciência pedagógica-participação (aqui com apenas $44 \%$ de educadores no grupo com envolvimento alto). Mais especificamente, entre $56 \%$ a $66 \%$ apresentaram alto envolvimento, isto é, superior à média na respetiva dimensão, como se pode observar na Tabela 1.

Tabela 1: Distribuição dos educadores de infância pelas dimensões do envolvimento, em termos de envolvimento baixo versus alto (valores em \%).

\begin{tabular}{ccc}
\hline Envolvimento & Baixo & Alto \\
\hline Vigor-dedicação (Vide) & 34 & 66 \\
Absorção (Abso) & 44 & 56 \\
Total da EAE-EI1 & 40 & 60 \\
Interpessoalidade (Inter) & 40 & 60 \\
\hline Consciência pedagógica-participação & 56 & 44 \\
(Copart) & & 59
\end{tabular}

Legenda: Total da EAE-EI1=Total da Escala de Avaliação do Envolvimento dos Educadores de Infância. 1;Total da EAE-EI2=Total da Escala de Avaliação do Envolvimento dos Educadores de Infância 2 .

Na revisão da literatura, não se encontraram estudos prévios, efetuados em Angola e com educadores de infância, que permitissem um contraste com os dados do estudo agora realizado. No entanto, comparados com estudos realizados noutros contextos, os resultados obtidos parecem ir no mesmo sentido, no que se refere à tendência de distribuição dos valores das dimensões nos níveis altos do envolvimento. Ainda que as amostras desses estudos não digam respeito a educadores de infância, os resultados obtidos nos mesmos são comparáveis aos aqui apresentados. Numa pesquisa realizada em Portugal por Picado (citado por Pocinho \& Perestrelo, 2011), com 400 professores do 1..$^{\circ}$ ciclo do ensino básico, que pretendia estudar o efeito da idade e do género no envolvimento e no burnout, confirmou-se que a dimensão Dedicação foi, das três dimensões do envolvimento, a que evidenciou valores mais altos, uma vez que a média de resposta foi de 5,83 (numa escala de Likert de 0 a 6 ) e a análise da distribuição das frequências mostrou que 97,2\% das respostas estava acima do valor central da escala de cinco pontos que foi utilizada. O autor verificou, também, que a dimensão vigor apresentou uma média de 4,87 sendo que $84,9 \%$ das respostas estavam acima do ponto 3 da escala, e que a dimensão absorção aparecia com uma média de 3,74 , com 58,9\% das respostas abaixo do ponto 4 da escala. Durán et al. (2005, citado por Pocinho \& Perestrelo, 2011), num estudo realizado em Espanha com professores do ensino básico, secundário e ensino de adultos, mostrou que os valores de envolvimento eram altos, com pontuações de média de resposta de 5,03 para a dimensão vigor, de 4,94 para a dedicação e de 4,71 para a absorção, numa escala de Likert de 0 a 6.

\subsection{Como se distribuem os educadores de infância pelas dimensões do autoconceito em termos de autoconceito baixo versus alto?}

Em resposta a esta questão de estudo, mais de metade dos educadores de infância apresentou autoconceito alto, na totalidade das dimensões do autoconceito, mais especificamente, entre $53 \%$ a $59 \%$, isto é, superior à média na respetiva dimensão. Em posteriores análises das diferenças entre o grupo com baixo e o grupo com alto autoconceito, não se registaram diferenças estatisticamente significativas. 
Deve atender-se à quantidade de educadores com baixo autoconceito, situada entre $41 \%$ e $47 \%$, com destaque na Competência, manifestamente com cuidados requeridos, como se pode observar na Tabela 2 .

Tabela 2: Distribuição dos educadores de infância pelas dimensões do autoconceito, em termos de autoconceito baixo versus alto (valores em \%).

\begin{tabular}{ccc}
\hline Autoconceito & Baixo & Alto \\
\hline Competência (Comp) & 41 & 59 \\
Relações-iniciativas-riscos & 44 & 56 \\
Autoaceitação (Auac) & 43 & 57 \\
Satisfação (Satis) & 46 & 54 \\
Total_EAA-EI1 & 47 & 53 \\
RPCL & 43 & 57 \\
PCCL & 47 & 53 \\
Total_EAA-EI2 & 47 & 53
\end{tabular}

Legenda: Total da EAA-EI1=Total da Escala de Avaliação do Autoconceito dos Educadores de Infância 1; RPCL=Reconhecimento Profissional em Contexto Laboral; PCCL=Pertençacooperatividade em Contexto Laboral; Total da EAA-EI2=Total da Escala de Avaliação do Autoconceito dos Educadores de Infância 2.

Quanto ao autoconceito, também não se encontraram pesquisas preliminares que possibilitassem uma comparação com os dados do estudo ora levado a efeito. Porém, comparados com um estudo realizado em contexto português, os resultados obtidos parecem algo similares, não obstante a amostra não ser de educadores de infância. Frade (2015) num estudo sobre motivação, envolvimento e autoconceito de militares dos cursos de formação de sargentos da marinha portuguesa, observou que uma maior percentagem de formandos $(60,1 \%)$ manifestava valores altos de autoconceito total, comparativamente à percentagem de formandos $(39,9 \%)$ que manifestava valores baixos nessa dimensão geral, destacando-se a dimensão Competência na qual se verificou que $66,7 \%$ dos formandos apresentava níveis altos nessa dimensão, relativamente aos 33,3\% que manifestava níveis baixos. Não obstante o estudo referido, a sua escassa contiguidade com o carácter desta pesquisa aborda uma noção que condiciona (de certa forma) a interpretação dos dados.

\subsection{Como se distribuem os educadores de infância pelas dimensões das necessidades de formação, em termos de interesse elevado, intermédio e nenhum interesse?}

Em resposta à esta questão de estudo, a maioria dos educadores de infância apresentou interesse elevado, em todas as dimensões das necessidades de formação. Mais especificamente, entre $59 \%$ e $99 \%$, isto é, superior à média na respetiva dimensão. Em posteriores análises das diferenças entre o grupo com nenhum, intermédio e elevado interesse, registaram-se diferenças estatisticamente significativas nas dimensões Gestão da profissão, Administração da progressão das aprendizagens, Utilização das novas tecnologias e no Total da EANF-EI. No entanto, também se registou na dimensão Utilização das novas tecnologias, $32 \%$ de educadores com interesse intermédio e $9 \%$ com nenhum interesse em frequentar ações de formação. De notar-se a quantidade de educadores com interesse intermédio e nenhum interesse, situada entre $32 \%$ e $9 \%$, como se pode observar na Tabela 3.

Tabela 3: Distribuição dos educadores de infância pelas dimensões da EANF-EI, em termos de interesse (valores em \%).

\begin{tabular}{|c|c|c|c|}
\hline Dimensões & NI & II & IE \\
\hline Gestão da profissão (Geprof) & 0 & 1 & 99 \\
\hline $\begin{array}{l}\text { Administração da progressão das } \\
\text { aprendizagens (Apap) }\end{array}$ & 1 & 8 & 91 \\
\hline $\begin{array}{c}\text { Utilização das novas tecnologias } \\
\text { (Unte) }\end{array}$ & 9 & 32 & 59 \\
\hline Total da EANF-EI & 0 & 3 & 97 \\
\hline
\end{tabular}


Relativamente às necessidades de formação, na revisão da literatura, não se encontraram igualmente estudos feitos em Angola e com educadores de infância, que permitissem uma comparação com os dados da pesquisa aqui realizada. Entretanto, comparados com os resultados de um estudo realizado em Portugal, sobre as necessidades de formação de professores do 1.ํ ciclo do ensino básico, apesar de os resultados irem em sentido algo diferente, podem ser de referência interessante. Rocha (2010) indicou na sua pesquisa que, a Utilização de recursos educativos digitais e a Utilização das potencialidades pedagógicas das novas tecnologias no ensino são áreas escolhidas pela maioria dos docentes como de muito e muitíssimo interesse, respetivamente.

\section{Conclusões}

Os dados descritos podem levar a pensar que, apesar das condições ainda instáveis do sistema educativo angolano, a maioria dos educadores de infância empenha-se no seu trabalho, tem uma perceção positiva de si próprio, das suas aptidões e competências, e do seu relacionamento com os outros, em contexto profissional e tem interesse elevado em gerir a profissão. No entanto, existe uma manifesta percentagem de educadores com baixo envolvimento, baixo autoconceito e com interesse intermédio em frequentar ações de formação na área das novas tecnologias.

Numa procura de explicação dos resultados encontrados em Angola, é provável que a percentagem de educadores com baixo envolvimento, situada entre $34 \%$ e $56 \%$, com destaque na Consciência pedagógica-participação, com baixo autoconceito, situada entre $41 \%$ e $47 \%$, com destaque na Competência e com interesse intermédio e nenhum interesse em frequentar ações de formação, situada entre $32 \%$ e $9 \%$, com destaque na Utilização das novas tecnologias, tenha a ver com a escassez de condições em geral e, em especial, condições promotoras de comprometimento e de bem-estar; impulsionadoras de habilidades, aptidões e melhoria das representações de si próprios em contextos laborais; estimuladoras de formação ao nível da operação e da utilização de meios informáticos, tudo isto em contextos de formação e de trabalho.

Tal deve ser visto como um forte apelo a mudanças que contribuam para a promoção do envolvimento, do autoconceito e dos meios para identificar, analisar e ajudar a suprir as necessidades de formação dos educadores de infância angolanos, quer ao nível da formação inicial como nos contextos de realização profissional e, divulguem a importância do uso das tecnologias de informação e comunicação ao longo do percurso docente. No entanto, este tipo de explicação encontra-se aberto a novos contributos, dada a carência de estudos prévios com proximidade à investigação (em Angola e com educadores de infância). Assim, sugere-se a realização de posteriores estudos, no sentido de uma comparação de dados.

\section{Referências}

Angst, Benevides-Pereira, \& Porto-Martins (2009). UWES - Utrecht Work Engagement Scale - Escala de Engagement no Trabalho de Utrecht. Versão brasileira.

Bailer, C. Tomitch, L. M. B., \& D'Ely, R. C. S. (2011). Planejamento como processo dinâmico: a importância do estudo piloto para uma pesquisa experimental em linguística aplicada. Revista Intercâmbio, XXIV, 129-146.

Barbier, J. M., Lesne, M. (1986). L'analyse des besoins en formation. Paris: Robert Jauze.

Borges, G. A. (no prelo). Envolvimento, autoconceito e formação: um estudo com educadores de infância em Angola. Tese de Doutoramento, orientada pelo Professor Doutor Feliciano H. Veiga, a apresentar no Instituto de Educação da Universidade de Lisboa, Portugal.

Dalfovo, M. S, Lana, R. A, Silveira, A. (2008). Métodos quantitativos e qualitativos: um resgate teórico. Revista Interdisciplinar Científica Aplicada, Blumenau. 2(4), 01-13. 
Fonseca, S. P. (2009). Autoconceito profissional dos professores de ciências e de matemática no 2. ${ }^{\circ}$ ciclo do ensino básico. Dissertação de Mestrado, Universidade de Lisboa, Portugal.

Pocinho, M. \& Perestrelo, C. X. (2011). Um ensaio sobre burnout, engagement e estratégias de coping na profissão docente. Educação e Pesquisa, 37(3), 513-528.

Rocha, S. C. V. G. (2010). Necessidades de formação de professores do 1. o ciclo do ensino básico - um contributo para o seu estudo. Tese de Mestrado, Instituto de Educação, Universidade de Lisboa, Portugal.

Saraiva, D. A. (2002). Participação dos professores na escola. Millenium, 25.

Veiga, F. H., Gonçalves, V., Caldeira, M. J., \& Roque, P. (2006). Representações dos professores acerca de si mesmos: adaptação portuguesa da escala "Teacher Self-concept Evaluation Scale". Actas do XIV Colóquio da AFIRSE. Para um Balanço da Investigação em Educação de 1960 a 2005. Teorias e Práticas (pp. 1131-1144).

Veiga, F. H. (2012). Transgressão e autoconceito dos jovens na escola (3. ${ }^{\mathrm{a}}$ Ed.). Lisboa: Fim de Século. 